\title{
LA PRODUCCIÓN CAPITALISTA DEL ESPACIO URBANO: DESAFÍOS PARA LA GEOGRAFÍA Y EL DISEÑO URBANO CAPITALIST PRODUCTION OF URBAN SPACE: CHALLENGES FOR GEOGRAPHY AND URBAN DESIGN
}

\author{
Diego Andrés González Cardona ${ }^{1}$ \\ María Isabel Lara Escobedo²
}

\section{Resumén:}

Los procesos actuales de financiarización inmobiliaria se basan en la especulación y en la expansión geográfica de las ciudades, moviéndose alrededor de tres factores: capital, tierra y trabajo.La ciudad es el epicentro de dicha expansión, correlacionándose directamente con la revalorización del uso del suelo y la creación de nuevos valores de cambio. Así mismo, el espacio geográfico es una representación social y es un proyecto en el que operan individuos, grupos sociales, instituciones y relaciones sociales, con sus propias representaciones y existe sólo a través de la existencia y reproducción de la sociedad, donde el Diseño Urbano tiene por objeto soportar el mundo del ser social, en toda su capacidad de definir lo que forme parte de su mundo real.a)Hoy por hoy, el espacio geográfico, al igual que el Diseño Urbano, se configuran por las dinámicas del sistema capitalista, es decir, por las relaciones de producción. El presente artículo se desplaza alrededor de varios supuestos: el espacio geográfico como construcción social; b)el capitalismo produce el espacio geográfico de acuerdo a sus necesidades para superar su principal contradicción interna: la sobreproducción; y c)el modo de producción capitalista realiza maniobras para estabilizarse y reproducirse. Se proponen, entonces, elementos para reflexionar sobre el compromiso social del Diseño Urbano, los procesos espaciales contemporáneos en cuanto a la plusvalía y la reorganización social y espacial, y sobre las pretensiones de convertir a las ciudades en espacios de consumo a través de la especulación financiera.

Palabras clave: ciudad - capitalismo - espacio geográfico - sobreproducción - financiarización.

\begin{abstract}
:
Current processes of financialization are based on real estate speculation and the geographical expansion of cities, moving around three factors: capital, land and labor. The city is the epicenter of this expansion, correlating directly with the revaluation of land use and the creation of new values change. Also, geographical space is a social representation is a project in which operate individuals, social groups, institutions and social relations, with their own representations and exists only through the existence and reproduction of society, where the Urban Design seeks stand the social world of being, in all its ability to define what part of your real world.Today, the geographical space

\footnotetext{
${ }^{1}$ Estudiante del Doctorado en Ciencias Sociales de la Universidad Autónoma del Estado de México - UAEM. Email: dagonzalezcardona@gmail.com

${ }^{2}$ Estudiante del Doctorado en Ciencias Sociales de la Universidad Autónoma del Estado de México - UAEM. Email: marybelbit@yahoo.com.mx
}

Revista de Direito da Cidade, vol.06, no02. ISSN 2317-7721 p. 369-386 369 
as well as Urban Design, are set by the dynamics of the capitalist system, namely, the relations of production. This article was moved around several assumptions: a) the geographical space as a social construction; b) Capitalism produces geographical space according to your needs to overcome its main internal contradiction: overproduction; and c) the capitalist mode of production makes maneuvers to stabilize and reproduce.Elements are then proposed to think about the social commitment of Urban Design, contemporary spatial processes regarding the plusvalue and social and spatial reorganization, and about the claims turn cities into spaces of consumption through financial speculation.

Keywords: city - capitalism - geographical space - overproduction - financialization.

Dicen los indios: ¿Que tiene dueño la tierra? ¿Cómo así? ¿Cómo se ha de vender? ¿Cómo se ha de comprar? Si ella no nos pertenece, pues. Nosotros somos de ella. Sus hijos somos. Así siempre, siempre. Tierra viva. Como cría a los gusanos, así nos cría. Tiene huesos y sangre. Leche tiene, y nos da de mamar. Pelo tiene, pasto, paja, árboles. Ella sabe parir papas. Hace nacer casas. Gente hace nacer. Ella nos cuida y nosotros la cuidamos. Ella bebe chicha, acepta nuestro convite. Hijos suyos somos. ¿Cómo se ha de vender? ¿Cómo se ha de comprar? Eduardo Galeno. Memorias del fuego (I)

\section{INTRODUCCIÓN}

La ciudad ha sido considerada como uno de los baluartes de la modernidad capitalista, pues es en ella en donde se ponen a circular los excedentes de la producción. En este sentido, se pueden identificar para el análisis tres grandes factores: capital, tierra y trabajo; de cuya interacción resultan categorías de análisis como capital acumulado, renta, beneficio e interés ${ }^{3}$. Por lo tanto, en el siguiente escrito se intentará reflexionar en torno a estos factores y su incidencia en los procesos actuales de financiarización inmobiliaria basada en la especulación y en la expansión geográfica de las ciudades.

En la búsqueda de conseguir este propósito, nos basaremos en lecturas más contemporáneas de la obra de Marx, resignificando la vigencia de sus análisis; como lo son las lecturas hechas por la Geografía Radical, encabezada por el geógrafo estadounidense David Harvey.

La tesis planteada es que tanto el espacio geográfico como el diseño urbano son construcciones sociales, al serlo, están configurados por las relaciones de producción, esto es, por

\footnotetext{
3 Para dicho análisis, se tomarán como referente las reflexiones económicas y filosóficas de Carlos Marx,
} principalmente expuestas en los Manuscritos de 1844 (MARX, 2001)

Revista de Direito da Cidade, vol.06, no02. ISSN 2317-7721 p. 369-386 370 
las dinámicas del capitalismo. Así, en vista de que el capitalismo está sustentado netamente en el crecimiento, requiere inminentemente de la expansión geográfica. Por lo tanto, la ciudad es el epicentro de dicha expansión en donde se revaloriza el uso del suelo y se crean nuevos valores de cambio.

\section{EL DISEÑO URBANO Y LA PRODUCCIÓN SOCIAL DEL ESPACIO GEOGRÁFICO}

La Geografía Radical ha desarrollado puntos de vista alternativos a las habituales evasiones tecnocráticas -cercanas a la apología del capitalismo- que dominan la geografía, en particular, y las Ciencias Sociales, más en general (HARVEY, 2007). Uno de los puntos centrales del análisis de los geógrafos radicales como Harvey, Soja, Peet y Santos ha sido el del desarrollo geográfico desigual. Para ello, se parte de la tesis de que el espacio geográfico es un producto social (LEFEBVRE, 2013; LACOSTE, 1973; SANTOS, 2000), un producto de la acción humana, y como tal, también un producto histórico. En otras palabras, el espacio geográfico es una representación social y es un proyecto, en el que operan individuos, grupos sociales, instituciones y relaciones sociales, con sus propias representaciones y proyectos. Es un producto social porque sólo existe a través de la existencia y reproducción de la sociedad.

En este sentido, siguiendo a Yves Lacoste (1973) es importante comprender que el espacio no es neutro ni inocente sino que es por excelencia uno de los campos de acción de las fuerzas políticas: el Estado. Así las cosas, el aparato de Estado es una entidad geográfica y organiza el espacio geográfico a modo de ejercer su poder sobre los hombres.

Teniendo en cuenta lo anterior, tenemos entonces que el espacio se convierte en un objeto más de diseño, por lo que hemos de contemplarlo en este sentido, estableciendo que:

1. No sólo se aborda el diseño a partir de las imágenes que se deberán construir; las denotaciones, como principio de orden, formarán parte de la propuesta, iniciando el proceso de sustanciación del objeto de diseño (Irigoyen, 2008: 55);

2. Cualquier elemento de opinión proveniente de la cultura, o de la ideología, debe aceptarse de inicio como componente para diseñar. Una opinión creída debe ser verificada para referir al objeto de diseño a través de sus propios atributos, los agentes que participan en él, los materiales empleados y, por supuesto, los usuarios; 
3. Las relaciones del objeto con el individuo son tan importantes como las de las partes del objeto entre sí, la escala y la proporción, están claras como factor de resolución en el diseño;

4. Toda actividad o trabajo, incluido desde luego el diseño, tiene por objeto soportar el mundo del ser social, otorgándole cualidades con su capacidad de definir objetos y hacer que formen potencialmente parte del mundo real. El diseño tiene como fin ofrecer entidades, principalmente de carácter objetual y luego material (Irigoyen, 2008: 56); un proyecto urbano o una obra arquitectónica se comprenden como entidades bien definidas, como totalidades relativas. Éstas finalmente son las que constituyen, junto con los hombres (los usuarios) en relación, el mundo de la cultura.

En esta misma dirección, Henri Lefebvre, que relacionó el espacio y su producción, en lo que llamó la reproducción de las relaciones sociales de producción, apuntó que el capitalismo se reproduce consumiendo espacio, o más concretamente que "sobrevive a través de la producción de espacio" (HARVEY, 2004: 99). Así el concepto de producción del espacio, se justifica al ser referido a un objeto de diseño en los contextos reales, en el campo de los conocimientos empíricos: sus determinaciones ambientales o culturales, la corroboración de sus significados y, principalmente, en cuanto a su sentido del ser y el estar.

El espacio geográfico como construcción social será, entonces, nuestro primer supuesto. Dicho espacio geográfico construido socialmente, responde permanentemente a las condiciones materiales de producción y apropiación de la riqueza, de lo cual resulta que el espacio es un valor culturalmente reconocido de la propiedad privada. De ahí la práctica capitalista de la eficiencia del diseño y uso del espacio: un número de $\mathrm{m}^{2}$ disponibles para la familia e instalaciones, en lo que toca a los servicios mínimos funcionales para la organización de la vida social, pero con la incapacidad de valorizar los elementos subjetivos de la experiencia cotidiana: exigencias espaciales, exigencias biológico-funcionales, exigencias psicológicas (comportamiento), donde el modo de uso del espacio conlleva un significado simbólico y donde, ante las condiciones materiales de producción y apropiación de dicho espacio, el usuario se convierte en su víctima.

Como cualquier otro recurso, el espacio es fuente de poder; y las modalidades de control de su uso, serán decisivas para hacer del recurso un instrumento de subordinación o de liberación, de diferenciación o de equidad. El modelo de utilización del espacio no responde en ninguna 
sociedad a un modelo funcional abstracto (necesidades) sino que más bien responde al modelo según el cual se configuran en cada sociedad las relaciones de poder.

El segundo supuesto, es que el capitalismo produce ese espacio geográfico de acuerdo a sus necesidades para superar su principal contradicción interna: la sobreproducción.

\section{LAS CONTRADICCIONES INTERNAS DEL CAPITALISMO}

La circulación del capital se puede considerar un proceso continuo en el que el dinero se usa para comprar mercancías (fuerza de trabajo y medios de producción, tales como materias primas, maquinarias, inputs energéticos, etc.); con el fin de combinarlas en la producción para producir una nueva mercancía que pueda venderse por el desembolso del dinero inicial más un beneficio (HARVEY, 2007: 332). En este sentido tendremos en cuenta diez puntos que HARVEY (2007) plantea como características fundamentales de la circulación del capital abordadas por Carlos Marx (2001):

1. La continuidad de la circulación del capital se basa en una continua expansión del valor de las mercancías producidas. Una economía capitalista "sana" es, por lo tanto, aquella que disfruta de una tasa de crecimiento positiva. Cuanto más nos acerquemos a una fase estacionaria, más enferma se juzga que está la economía. Esto se traduce en una ideología del crecimiento ("el crecimiento es bueno") sin importar las consecuencias medioambientales, humanas y geopolíticas;

2. El crecimiento se alcanza mediante la aplicación de trabajo vivo a la producción, el cual es la fuente exclusiva del valor real añadido en la producción;

3. El beneficio tiene su origen en la explotación del trabajo vivo en la producción. Marx (2001) lo planteaba como un proceso de enriquecimiento progresivo en el cual el Capital es la acumulación de trabajo arrebatado al obrero a partir de que al aumentar la división del trabajo aumenta el número de obreros, es decir, aumenta el número de fuentes de trabajo que será arrebatado;

4. La circulación de capital se basa en una relación de clase. La circulación de capital supone la compra y la venta de fuerza de trabajo como mercancía. La separación entre vendedores y compradores abre una relación de clase entre ellos. Sin la relación capital-trabajo 
expresada mediante la compra y la venta de fuerza de trabajo no podría haber explotación, ni beneficio, ni circulación de capital;

5. Esta relación de clase implica oposición, antagonismo y lucha. Hay dos cuestiones relacionadas en juego: cuánto tienen que pagar los capitalistas para obtener los derechos de utilización de la fuerza de trabajo y qué comprenden exactamente esos derechos. Los enfrentamientos por la tasa salarial y las condiciones de trabajo (la duración de la jornada laboral, intensidad del trabajo, control sobre el proceso de trabajo, etc.) son en consecuencia endémicos a la circulación del capital;

6. El modo de producción capitalista es tecnológicamente dinámico. El impulso de crear revoluciones perpetuas en la productividad social del trabajo radica, en un principio, en las fuerzas gemelas de la competencia intercapitalista y la lucha de clases;

7. El cambio tecnológico y organizativo exige inversión de capital y fuerza de trabajo;

8. La circulación del capital es inestable. Personifica contradicciones fuertes y perjudiciales que lo hacen tender crónicamente a la crisis, a partir de una contradicción básica: el sistema tiene que expandirse mediante la aplicación de trabajo vivo a la producción, mientras que la principal senda del cambio tecnológico es suplantar al trabajo vivo en la producción, el verdadero agente de la expansión. El antagonismo subyacente explota periódicamente en crisis totales de acumulación, de alteraciones completas del proceso de circulación de capital;

9. La crisis se manifiesta en general como una situación en la que los excedentes de capital y de trabajo que el capitalismo necesita para sobrevivir ya no pueden absorberse: sobreacumulación. Este es el tipo de irracionalidad, con enorme capacidad productiva inutilizada y elevado desempleo en la que la mayoría de las economías occidentales se han hundido estos años pasados (por ejemplo la situación de España con más de 6 millones de desempleados). A este respecto Marx (2001) siguiendo a Adam Smith, identificaba cómo, no sólo aumenta el volumen de actividad en los países con el crecimiento del capital que en ella se emplea, sino que, como consecuencia de este aumento, un mismo volumen industrial produce mucha mayor cantidad de obra;

10. Los excedentes que no se pueden absorber se devalúan, a veces incluso se destruyen físicamente. El capital puede devaluarse en forma de dinero (inflación o impago de deudas), 
en forma de mercancías (existencias no vendidas, ventas por debajo del precio de coste, desperdicio físico), o en forma de capacidad productiva (instalaciones productivas inutilizadas o infrautilizadas).

Estos diez puntos constituyen contradicciones al relacionarse entre sí. Resaltamos la contradicción entre crecimiento, innovación tecnológica y trabajo vivo, manifestada en los puntos 8,9 y 10. El cambio tecnológico aumenta la producción favoreciendo el crecimiento, pero anulando la fuerza de trabajo vivo (desempleo) que es la base del valor y agotando las fuentes de materias primas. Así, se llega a un punto de sobreproducción (mercancías en stock que representan trabajo acumulado, es decir, trabajo muerto) que no es consumida debido a que no hay poder adquisitivo, por parte de las masas, para hacerlo. Existe por lo tanto una anulación entre los principios rectores de la economía capitalista, procurando una situación en donde el crecimiento se plantea como infinito en un mundo de recursos finitos.

Sin embargo, ¿qué maniobras se realizan para estabilizar el modo de producción capitalista? ¿Cómo se soluciona el problema de las materias primas y de la absorción de las mercancías sobre-producidas? Por un lado tenemos lo que David Harvey (2007) llama la solución espacial y, por otro lado, el aumento de créditos: la financiarización.

\section{LA CIUDAD: SOLUCIÓN ESPACIAL Y LA PRODUCCIÓN CAPITALISTA DEL ESPACIO}

El capitalismo está siempre produciendo excedentes en forma de ganancia, teniendo como consecuencia un proceso permanente de crecimiento que genera a su vez un exceso de liquidez que requiere ser absorbido. El capitalismo, entonces, se reproduce consumiendo espacio e introduce un nuevo término para determinar la relación entre el sistema capitalista y el espacio: el ajuste espacio-temporal (Harvey, 1982; 2004; 2007). Este arreglo consiste en dos mecanismos espaciales para salvar las crisis de sobreacumulación: la expansión geográfica y la reorganización espacial, es decir, la producción de espacio ya sea por crecimiento o por transformación. Se trata de utilizar intensivamente el circuito secundario de acumulación capitalista, el espacio construido, para resolver las crisis capitalistas. Ya Hegel en la "Filosofía del Derecho" presentaba el imperialismo y el colonialismo como posibles soluciones a las contradicciones internas de lo que él considera una sociedad civil "madura" (HARVEY, 2007:392) Estos mecanismos - el ajuste espacio temporal para salvar las crisis capitalistas y la estructuración del espacio como estabilización social - demuestran 
la clara relación que existe entre el desarrollo capitalista y la producción de espacio, en sus versiones de transformación y crecimiento.

Además de la escala de los procesos de urbanización, destaca el surgimiento e intensificación de algunos procesos de transformación urbana en el interior de las ciudades. Entre los procesos espaciales contemporáneo destacan los mecanismos de gentrificación o elitización, en cuanto a la generación de la plusvalía por la reorganización social y espacial y la museificación y tematización, en cuanto a la intervención y reorganización espacial para convertir a las ciudades en objeto del turismo masificado, es decir, en espacios de consumo.

En primer lugar, la gentrificación o elitización (DELGADO, 2011) consiste en un proceso de transformación urbana en el que la población de un barrio deteriorado es progresivamente desplazada por otra de un mayor nivel adquisitivo a la vez que éste se renueva. De esta manera, se concibe el proceso de elitización con base en tres fases dentro del ciclo de vida de un sector urbano. Este ciclo de vida es el que han llevado los barrios industriales tradicionales de ciudades occidentales que se encuentran en desarrollo y expansión en el periodo posfordista.

a) Nacimiento: La primera fase del ciclo vital que conduce a un sector urbano a la elitización es su configuración como territorio obrero en la ciudad capitalista moderna. La industrialización creó una configuración del centro urbano o como parte del mismo por la presencia de los obreros y de la producción, al mismo tiempo al crear nuevos territorios industriales que pueden surgir sobre barriadas antiguas de la ciudad o sobre territorios vacíos demográficamente, se activa el valor de cambio de nuevas zonas suburbanas hacia donde se desplazan las clases altas.

Así, la industrialización supone la atracción de grandes oleadas de inmigración provenientes de zonas rurales. Los nuevos pobladores que vienen a trabajar en las industrias, situadas en el centro de la ciudad y en las zonas portuarias, buscan estar cerca al lugar de trabajo, por lo cual el barrio obrero da lugar a un tipo particular de urbanismo y a una serie de relaciones sociales propias: tipos de vivienda, talleres, tiendas y costumbres diferenciadas de los barrios de las clases altas; todo un diseño urbano sui generis.

Por otro lado, la evolución de los transportes (trenes, tranvías) permitió en las ciudades modernas la expansión de las clases altas hacia la periferia, hacia zonas suburbanas. La zona alejada supone para las clases altas grandes espacios y tranquilidad, frente a la densa masificación y la 
insalubridad del centro industrial moderno. Esto configura un modelo espacial de división social y de desarrollo geográfico desigual entre las clases altas y la clase trabajadora. Así las cosas, en las ciudades comienzan a desarrollarse fenómenos especulativos de acuerdo a la inmigración obrera y al aumento en la demanda de hospedajes; de ahí que la especulación y la producción de la vivienda como mercancía en las ciudades se convierte en un importante factor de acumulación del capital.

Este proceso tiene varias consecuencias. El orgullo del Capital, de la especulación, es ofrecer ahora grandes oportunidades para "la casa propia", pero, no se incluye en la "oferta" el significado social de ésta: la casa propia, donde ya no se trabaja, no se juega, no se convive; ya ahí, no se nace ni se muere, no se festeja ni se divierte, no se cría a los hijos ni se cocina: todo esto se hace afuera, la casa es apenas un refugio, un escape nocturno del mundo hostil.

Si en el trabajo, en el aprecio de los demás, en el ejercicio auténtico del poder, los niveles más altos, y muy frecuentemente también los intermedios, permanecen cerrados; por lo menos la casa (y lo que ésta contiene y las formas de usarla) deberían restituir la ilusión del éxito alcanzado, de la insertación obtenida, de la igualdad con los vértices; pero, el ideal moral obrero, dicta:

"Cuanto menos comas y bebas, cuantos menos libros leas, menos vayas al teatro, al baile y a la taberna, menos pienses, ames, teorices, cantes, pintes, hagas versos; más ahorrarás, mayor será tu tesoro, que no comerán la polilla ni el polvo, mayor será tu capital".

No se trata entonces sólo de producir, sino de reproducir; es la Economía Política, la ciencia de la riqueza, de la abstinencia, del ayuno, del ahorro, hasta ahorrar al hombre incluso, la necesidad de aire puro o de movimiento físico, que expresa solamente a su modo las leyes morales: canasta básica, vivienda de interés social, mobiliario pequeño para adecuarse al espacio; en fin, el "mínimo" ¿calculado por quién?

Descapitalización: la segunda fase es la descapitalización y abandono del sector, puesto que es necesario que exista una etapa de descapitalización y desvalorización de los sectores antes obreros, lo que permite las grandes diferencias entre el valor de la tierra (valor comercial) de la zona degradada y el valor potencial (valor de uso) que por su ubicación podría tener, reforzado por los servicios que se convierten en el plus: vías, transporte, cercanía a centros comerciales, colegios, hospitales, parques, etc. La degradación de las condiciones de vivienda, fruto del retiro de inversión, provoca un efecto de rechazo sobre los habitantes del barrio, que ante las limitaciones en

Revista de Direito da Cidade, vol.06, no02. ISSN 2317-7721 p. 369-386 377 
su poder adquisitivo irán desplazándose paulatinamente a otras áreas de la ciudad ${ }^{4}$. A medida que el deterioro de los sectores avance, el abandono acabará expulsando a la mayoría de los habitantes históricos; en este momento es cuando queda disponible para ser elitizado.

b) Revalorización: debemos tener en cuenta que las dinámicas espaciales del diseño urbano cambian teniendo en cuenta el modelo económico de desarrollo que tenga vigencia. Partiendo de esto, podemos evidenciar que luego de la crisis estructural del sistema económico global de los años setenta donde se empieza a dejar de lado el modelo de la producción en cadena, entramos en lo que se ha llamado la era post-fordista en occidente. De ahí que los centros de las ciudades pasan a ser fundamentales para el desarrollo económico del sistema, ahora como centros financieros, de servicios y de gobierno, nodos de comunicación con ciudades y empresas donde se realizan los negocios más rentables. La oferta de los centros turísticos, servicios comerciales, financieros y culturales dan un gran valor a la mercancía inmobiliaria, que se encarece más y más en los centros de las ciudades, siendo atractivo solo para las clases con mayor poder adquisitivo.

En segundo lugar, la museificación puede ser entendida como el embalsamamiento en su estado histórico de una ciudad o una parte de ella, limitando sus propias dinámicas con la finalidad de hacer atractivo ese espacio como objeto de consumo. Igualmente, la tematización consiste en:

Dedicar partes acotadas, comprensibles y completas de ellas mismas a un tema concreto, que se ofrece como atractivo al turismo local e internacional [...] lo que implica simplificar la complejidad de la propia historia para ofrecer un discurso simple y transmisible. (DELGADO, 2011)

A partir del análisis de estos dos procesos expuestos anteriormente, elitización y museificación, podemos observar también que el proceso de legitimación de los objetos de diseño depende de su capacidad para insertarse en determinados campos sígnicos de la cultura. Esa capacidad, para un mismo objeto, se aplica en distintos niveles y puede dar por resultado diferentes tipos de inserción en la cultura. Un objeto idóneo para el medio rural no lo es tanto para el medio urbano, sin embargo, si un objeto no se ajusta o no es aceptado, se adecua al medio que lo va a

\footnotetext{
${ }^{4}$ Sin embargo hay que tener en cuenta que se han evidenciado experiencias de diferentes proyectos comunitarios de resistencia ante las constructoras y empresas inmobiliarias para evitar el desplazamiento de los habitantes históricos.
}

Revista de Direito da Cidade, vol.06, no02. ISSN 2317-7721 p. 369-386 378 
recibir. La flexibilidad y la convertibilidad de su naturaleza, ajusta o cambia relativamente, sus principios (Irigoyen, 2008: 165).

Es indudable que en el diseño se busque, entre otras cosas, la forma como resultado, pero no puede establecerse como el medio para transformar la realidad. No podemos abandonar las causas de los fenómenos del diseño consideradas como contenidos y sustancias; pensar que lo verdaderamente importante es la forma de los espacios es potenciar al efecto como causa (Irigoyen, 2008: 120). Si bien los espacios pueden surgir y desaparecer, experimentar diversos cambios y transformarse, la sustancia homogénea e invariable es su base (es en donde nos movemos), y sólo sería modificable por su exterioridad formal. La morfología es, en todo caso, el ordenamiento del proceso de su intelección. Se trata de una condición relacional, donde la cosa (el espacio) no puede reducirse a su tratamiento.

Decir que el diseñador es soporte de las relaciones sociales que le corresponden en la división técnica y social del trabajo no es reducirlo a instancia pasiva, o puramente mecánica. No se puede suponer que las prácticas del diseño no responden a determinadas regularidades sociales, regularidades que se producen ciertamente al margen de la voluntad y la conciencia de los individuos.

En realidad, lo social manifiesta dos niveles o modos de ser, presentes e ineludibles en el diseño: Primero, las estructuras sociales externas, lo social hecho cosas (la ciudad por ejemplo, si el referente es empírico) o bien, determinados campos de posiciones sociales que se han constituido en dinámicas históricas particulares: el sistema escolar, el campo económico, el campo político. Segundo, las estructuras sociales internalizadas, incorporadas al diseñador como agente social del diseño, en forma de esquemas de percepción, pensamiento y acción: los habitus (Irigoyen, 2008: 86).

En este sentido, no podrá existir el lugar de nadie o de nada; los lugares son siempre de alguien y para algo. Las formas de relación social serán consideradas por la manera en que se dan esos procesos sociofísicos de transformación que determinan históricamente la naturaleza social del lugar.

El mundo no sólo se comprende porque es interpretado: se entiende y comprende porque se puede leer. Los automóviles, los semáforos, la ropa o los edificios, dicen muchas cosas sobre nosotros y los demás. La lectura de los objetos diseñados permite comprender la cultura que les da 
arraigo y estudiar las herramientas con las que se hicieron. En mayor medida, permite interpretar las relaciones que debieron mantener los individuos para realizar esos objetos. En efecto, la manera en que se dan las relaciones, pone al ser humano en condiciones de interpretar ese mundo de una forma determinada, como es percibido (Irigoyen, 2008: 331).

La socialización en el diseño puede definirse como la inducción amplia y persistente, en el mundo objetivo de la sociedad, de todos aquellos que individual y colectivamente se encuentran orientados por la realización de los objetos que darán soporte material a la cultura en su conjunto. Es un proceso de articulación creciente, de enorme complejidad, por el cual los agentes productivos, a lo largo de su vida, se convierten en miembros de la sociedad al adquirir el conocimiento específico de determinados roles, lo que significa la internalización de campos semánticos que estructuran tanto las interpretaciones de los objetos, como los comportamientos de rutina necesariamente institucionalizados que los involucran (Irigoyen, 2008:144).

Se inventa que se practica la libertad de elegir entre todo lo dado; esa es la identidad que mejor se acomoda a una sociedad como la actual. Toda referencia al consumismo no es ni mera coincidencia ni surge gratuitamente. En el fondo, es el modelo de libertad imperante que caracteriza a toda sociedad neoliberal (Irigoyen, 2008: 94). Esa misma libertad real y relativa es la que hoy, históricamente, establece límites a las especulaciones del diseño. La libertad social plena, a la que todos podrían aspirar, ésa que ni siquiera se ha podido concretar como idea, la equitativa, no pondría límites al diseño, pondría los límites en la apropiación y producción capitalista del espacio.

El espacio no es un contenedor homogéneo ni tampoco una abstracción geométrica. Es diferente estar aquí o estar allá, hay espacios buenos y espacios malos, espacios en los que se está cómodo y otros donde el malestar se hace presente. El espacio del que cada quien (individuo, grupo, clase social) dispone concretamente en una sociedad determinada, refleja en mucho su ser: libertad de acción, de entrar, de salir, de pasar por. En cada sociedad, la relación con el espacio refleja al sistema.

En todo este proceso juegan un papel importante los Planes de Ordenamiento Territorial POT- en el sentido que se construyen a partir de evaluaciones permanentes de las dinámicas territoriales en cuanto al uso del suelo y del beneficio que se puede obtener de él.

Revista de Direito da Cidade, vol.06, no02. ISSN 2317-7721 p. 369-386 380 
Así mismo, las políticas públicas, en tanto mecanismos emergentes para enfrentar problemas públicos que afectan valores sociales, incluyen el abordaje de las ineficiencias de la gestión pública en todos los niveles de gobierno que no permiten que los problemas sean resueltos. Como consecuencia de ello: “...las políticas públicas se convierten en intervenciones sistemáticas que deben contribuir en el mejoramiento de los problemas, en su eliminación si ello fuera posible, o en su anticipación o prevención" (González, 2002: 28).

Sin embargo, las políticas urbanas practicadas hasta ahora en ciudades como Toluca, México, parecen desfasadas, orientadas tradicionalmente a la centralización y con poca o carente capacidad de reacción, lo que se refleja en la poca duración de los cambios, leves o radicales, que en muy poco tiempo son repintados, reencarpetados, reubicados, demolidos o simplemente, desaparecidos.

Para Marc Augé, la sobremodernidad contiene tres figuras de exceso:

a) La superabundancia de acontecimientos;

b) La superabundancia espacial;

c) La individualización de las referencias.

Estas tres características encuentran naturalmente su expresión completa en los llamados no lugares (Augé, 2005: 112).

La sobremodernidad convierte a la historia en una curiosidad, hace de lo antiguo un espectáculo específico, al igual que torna lo exótico y lo particular de toda localidad en un no lugar marcado en las guías para el viajero y desconocido para los habitantes cotidianos que se mueven en su propia ciudad.

Los individuos se mueven en la ciudad y, en un momento dado, se acercan haciendo lo social y disponiendo los lugares. El espacio de la sobremodernidad trabaja en contradicción: tiene que ver con individuos (burócratas, estudiantes, paseantes) pero no están localizados, identificados ni socializados, más que a la entrada o a la salida.

Si los no lugares son el espacio de la sobremodernidad, es necesario explicar esta paradoja: el juego social parece desarrollarse fuera de los puestos de avanzada de la contemporaneidad (Augé, 2005: 114).

Frecuentemente se escucha(o se comenta) que la ciudad es un caos, pero los no lugares acogen a los individuos cada día más numerosos. El usuario de los espacios en la ciudad, resulta ser 
la presa favorita de quienes tienen como máximo propósito, el territorio a conquistar. En definitiva, el no lugar es lo contrario de la utopía: existe y no postula ninguna sociedad orgánica (Augé, 2005: 114).

Los no lugares, son, hoy por hoy, un componente esencial de toda existencia social. En sus modalidades más limitadas, o en sus expresiones más exageradas, es la experiencia del no lugar (indisociable de una percepción más o menos clara de la aceleración de la historia y del achicamiento del planeta).

¿Cómo pensar los problemas de cualquier ciudad? Cualquier búsqueda, cualquier secuencia, cualquier tránsito por el tiempo y el espacio puede ser concebido como laberíntico en la medida en que presenta obstáculos, genera digresiones y posibilita la iteración involuntaria de quien efectúa el recorrido (Zavala, 1999: 61).

La perspectiva de la metáfora laberíntica se ubica en los trazos de calles de la ciudad, en los encuentros de los usuarios, en la manera en que la ciudad, propicia en sus habitantes una determinada manera de emplear el tiempo y hacer suyo el espacio.

Se trata de entender la amplitud de la función social del diseño a partir de su replanteamiento: una realidad social como objeto de diseño. La comprensión de los productos de trabajo como propuesta creativa y posiblemente transformadora, amplía la posibilidad de reentender los niveles de su compromiso social.

El concepto de espacio no es ideológico por sí mismo. Son los individuos quienes por el modo de uso de dichos conceptos, otorgan tal sentido, reconociéndolo como un producto secuenciado de la relación entre lo social con la redundante naturaleza del medio ambiente (Irigoyen, 2008: 309).

Por ello, nuestro espacio, es el espacio inherente y se caracteriza porque posee determinada condición biográfica. Vida concreta y vivienda, (o lugar en que nuestra vida se localiza y establece), aparecen, directamente asociadas, y requieren una consideración rigurosa y plena en cuanto a cuáles son las condiciones en que la vida humana tiene lugar, y qué actividades necesitamos emprender para dar localidad y ocasión a nuestra inalienable manera de vivir.

Teniendo en cuenta las características de estos procesos, podemos comprender la relación entre Urbanismo y desigualdad social (ver HARVEY, 1992) basada en la concentración geográfica de un producto social excedente, esto es la tierra y el uso del suelo, lo cual es el plusvalor. La 
extracción de este plusvalor se da a partir de la especulación en los derechos de propiedad y de los intereses de los créditos financieros para adquirir vivienda; es decir, se crean "supuestos" nuevos beneficios que incrementan el valor de cambio de la vivienda (cercanía a centros de servicio, innovación arquitectónica, vista panorámica, dotaciones como gimnasio, piscina, salón de juegos, etc.) y se amplían los cupos de crédito para vivienda.

Con estas dos últimas estrategias, se solucionan los dos límites del capitalismo: el espacial y el temporal. El espacial en cuanto se revaloriza la tierra y el uso del suelo a través de la recapitalización y del crecimiento vertical de la vivienda, (ya no horizontal como sería en el siglo XIX). El temporal, en tanto que si el problema de la sobreproducción es la generación de excedentes tanto de mercancías como de mano de obra que no pueden ser consumidos recíprocamente, ya que si hay mucha producción no hay empleo y al no haber empleo no hay poder adquisitivo, (anulándose así la fuente del capital que es el Trabajo Vivo). El crédito elimina el espacio mediante el tiempo (HARVEY, 2007), es decir, que el crédito contrae la distancia entre tiempo y espacio ya que lo que se hace a través del crédito es una promesa de venta de fuerza de Trabajo Vivo a futuro para poder consumir lo que se produjo y se acumuló en el pasado. Sin embargo, esto resultaría ser una solución temporal, ya que lo único que representa es un desplazamiento temporal de la crisis hacia el futuro.

\section{LA FINANCIERIZACIÓN Y LAS BURBUJAS INMOBILIARIAS}

La burguesía construye una sociedad a su imagen y semejanza. Esta frase, expresada en el "Manifiesto del Partido Comunista", refleja las circunstancias bajo las cuales las ciudades son el foco de la puesta en marcha de los proyectos modernizadores de la burguesía. Los proyectos de urbanización que recapitalizan el uso del suelo, evidencian las estrategias que se crean para que las mercancías, convertidas en trabajo muerto, vuelvan a circular para aumentar el valor de cambio.

Retomamos entonces los conceptos de Beneficio, Renta e Interés. Beneficio puede ser entendido como el resultado final de la circulación del dinero intercambiado por una mercancía que ha sufrido alguna transformación lo que ocasiona que se obtenga al final una suma mayor de dinero a la invertida inicialmente (D-M-M'-D'). Por Renta, Marx (2001:38) siguiendo a Smith plantea que puede considerarse como producto de la fuerza natural cuyo aprovechamiento arrienda el propietario al arrendatario. Este producto es mayor o menor según sea mayor o menor el volumen

Revista de Direito da Cidade, vol.06, no02. ISSN 2317-7721 p. 369-386 383 
de esta fuerza, o en otros términos, según el volumen de la fertilidad natural o artificial de la tierra. Finalmente por Interés entendemos el dinero que se obtiene de la utilización del dinero (D-D'), lo que hace que no haya, a diferencia del beneficio, trabajo vivo como sustento de esta ganancia.

Teniendo en cuenta lo anterior, se puede ver cómo la especulación financiera ha estado guiada por un enriquecimiento basado en el interés, mas no en el beneficio, lo que hace que no haya sustento material de su crecimiento. Por ello, las cifras de ganancia presentadas por los bancos en sus informes anuales muestran crecimientos exponenciales. Así mismo, el aumento exponencial en el precio de la vivienda es reflejo de este proceso.

\section{A MANERA DE CONCLUSIÓN}

A partir de lo expuesto en los renglones anteriores, podemos ver que la construcción del espacio se hace a favor del sistema imperante, con el fin de alcanzar un mayor control poblacional a través de una estrategia fundamental: el desplazamiento, que reconfigura constantemente la división entre "civilizados y bárbaros". Así entonces, vemos cómo la urbanización se convierte en el motor para la modificación y expansión en la ciudad y lograr la expulsión de los pasivos del espacio creando nuevas fuentes de creación especulativa del dinero que a largo plazo se refleja en las crisis de los sistemas financieros. Ante estas situaciones, se hace cada vez más vigente los análisis desde la teoría marxiana respecto a las dinámicas del capital.

Es esta otra gran contradicción, porque el diseño urbano deja de actuar de acuerdo a su naturaleza: como el dispositivo para lograr en la ciudad un espacio de calidad y convivencia, un espacio que se disfruta, que se recorre sin temor y sin queja. Un diseño que busca reducir los desórdenes que impiden elevar la calidad de vida de la ciudadanía, un diseño donde la arquitectura se involucra como campo de conocimiento con una participación clara en el proceso de desarrollo social y donde el diseño urbano garantice la conformación y el desplazamiento de la dinámica sociourbana, pero que, para la producción capitalista del espacio, es un grave peligro.

Lo necesario es restituir al hombre una identidad propia, que lo aleje del actual proceso de enajenación que se está verificando en nuestras ciudades, para volverlo a conducir al verdadero significado de habitar para hacerlo capaz de apreciar las cosas por su valor, en vez de considerarlas simplemente como objetos de uso y de propiedad privada. (Coppola, 2004)

Revista de Direito da Cidade, vol.06, no02. ISSN 2317-7721 p. 369-386 384 


\section{BIBLIOGRAFÍA}

AUGÉ, M. (2005), Los "no lugares" espacios del anonimato. Una antropología de la sobremodernidad. Gedisa, España.

COPPOLA PIGNATELLI, P. (2004), Análisis y diseño de los espacios que habitamos. Pax. México.

DELGADO JIMÉNEZ, A. (2011), De la crisis fordista a la crisis de la financiarización, Polis [En línea], 28 I, Puesto en línea el 15 abril 2012, consultado el 24 de octubre de 2013. URL: http://polis.revues.org/1342; DOI: 10.4000/polis.1342

GONZÁLEZ, M (2002), Desarrollo y Uso de Modelos de Políticas Públicas: análisis de la Experiencia Docente en el Posgrado de Ciencias Políticas de la USB (1998-2001). Universidad Simón Bolivar, en "Perfiles". Revista de investigación Educativa. Año 23, No.1. Caracas.

HARVEY, D (1990), Los límites del capitalismo y la teoría marxista. México: FCE. (1992), Urbanismo y desigualdad social. España: Siglo XXI. (2004), El "nuevo" imperialismo: acumulación por desposesión". En: Pantich, Leo \& Colin (2007), Espacios del capital: hacia una geografía crítica. Madrid: Akal.

LEYS (ed.), El Nuevo desafío Imperial, (pp. 99-129). Buenos Aires: Merlin Press - CLACSO.

IRIGOYEN CASTILLO, J.F. (2008), Filosofía y diseño: una aproximación epistemológica. México: UAM-Xochimilco.

LACOSTE, Y (1973), La Filosofía de las Ciencias Sociales. París: Hachette.

LEFEBVRE, H (2013), La producción del espacio. Madrid: Capitán Swing. ISBN: 9788494169052

MARX, C (2001), Manuscritos económicos y filosóficos de 1844. Biblioteca Virtual Espartaco. SANTOS, M (2000), La naturaleza del espacio. España: Ariel.

ZAVALA, L (1999), La precisión de la incertidumbre: posmodernidad, vida cotidiana y escritura. México: UAEMéx. 
Revista de Direito da Cidade

Trabalho enviado em 01 julho de 2014.

Aceito em de 20 setembro de 2014. 